\section{FDA approves first digital pill}

The FDA approved a first drug-device product that uses a digital chip to monitor ingestion. This tracking technology could help address the costly problem of poor drug adherence in both medical practice and clinical trials, if it lives up to its promise (Nat. Rev. Drug Discov. 14. 735-737; 2015).

Otsuka's Abilify MyCite combines the drug developer's atypical antipsychotic aripiprazole with Proteus Digital Health's tracking device - a $1 \mathrm{~mm}$ ingestible chip that is activated when it comes into contact with stomach acid, sending a signal to a wearable band-aid sized patch, which is paired to a smartphone. The agency approved the product for schizophrenia, bipolar I disorder and depression.

The agency first approved aripiprazole for schizophrenia in 2002. It approved Proteus's device in 2012, but only as a stand-alone device that pharmacists could combine with active drugs using an 'over-encapsulating' capsule. Otsuka first submitted Abilify MyCite for approval in 2015, but the agency rejected it the following year. Otsuka resubmitted the product earlier this year.

The approval now provides "an innovative way to provide individuals with serious mental illness, and selected members of their families and care teams, with information on objective medication-taking patterns to help inform the patient's illness management and personalized treatment plan," says John Kane, psychiatrist at Northwell Health and investigator on Proteus-sponsored trials.

The FDA noted that the sponsors have not shown that the product improves treatment adherence.

Drug non-adherence is thought to be responsible for up to US $\$ 300$ billion in avoidable health-care costs, including unnecessary hospitalizations, nursing home admissions and premature deaths. If Otsuka and Proteus can prove that their product does improve adherence, tracking technology will likely be paired with other drugs as well.

Drug developers are also interested in using tracking technologies in clinical trials, to ensure that trial participants take their drugs properly and to make it easier to interpret trial results. AiCure, another firm that is active in this space, uses video facial recognition to monitor adherence during clinical trials.

Asher Mullard

\section{Second anticancer CAR T therapy receives FDA approval}

The FDA's approval of Gilead Sciences and Kite Pharmaceutical's axicabtagene ciloleucel for relapsed or refractory large B cell lymphoma in October marked the second green light for the emerging chimeric antigen receptor (CAR) T cell modality. In August, the agency approved Novartis's tisagenlecleucel for acute lymphoblastic leukaemia.

CAR Ts are ex vivo gene therapies, in which $T$ cells are removed from cancer patients, genetically modified to express cancer-seeking receptors, and then re-infused back into patients. Both axicabtagene ciloleucel and tisagenlecleucel are modified so that the engineered immune cells can target the B cell antigen CD19, leading to the depletion of normal and malignant $B$ cells for the treatment of various blood cancers.
The approval of axicabtagene ciloleucel was based on a 101-patient pivotal trial, which showed third-line use of the therapy resulted in an objective response rate of $72 \%$ and a complete remission rate of $51 \%$ in patients with large B cell lymphoma. The treatment carries a black box warning noting the risk of cytokine release syndrome and neurological toxicities. Novartis's tisagenlecleucel, which the company recently submitted for regulatory approval in the large B cell lymphoma setting, also carries these warnings on its label.

The field is working to mitigate toxicity risks for next-generation CAR Ts, and to figure out how to use the modality in solid tumours (Nat. Rev. Drug Discov. 16, 301-304; 2017).

Gilead acquired Kite in August for US $\$ 12$ billion. Analysts forecast that the newly approved CAR T could achieve sales of more than $\$ 3$ billion by 2023, according to Clarivate Analytics' Cortellis database. The company has two other engineered $\mathrm{T}$ cell therapies in its clinical pipeline: a phase I CAR T that targets BCMA, for multiple myeloma, and a phase I engineered T cell receptor product that targets MAGEA3 and MAGEA6, for solid tumours.

Asher Mullard

\section{BTK inhibitors get a boost}

The FDA granted accelerated approval to AstraZeneca and Acerta Pharma's acalabrutinib for mantle cell lymphoma, a second approval for the BTK inhibitor class. The agency approved AbbVie and Janssen's first-in-class BTK inhibitor, ibrutinib, in 2013 for the same indication. Ibrutinib has since picked up supplementary approvals in a few settings, including chronic lymphocytic leukaemia and graft-versus-host disease.

The BTK kinase acts downstream of B cell receptor signalling, and is implicated in the activation of several cell-survival pathways that are often co-opted by lymphoid malignancies (Nat. Rev. Drug Discov. 12. 229-243; 2013).

In a 124-patient pivotal trial of acalabrutinib in mantle cell lymphoma, AstraZeneca and Acerta showed that second-line use of their drug resulted in complete or partial responses in $80 \%$ of patients.

Ibrutinib currently earns more than US $\$ 2.8$ billion per year in annual global sales, and analysts forecast it could reach close to $\$ 9.5$ billion by 2021. Analysts forecast sales of just over $\$ 1$ billion for the newly approved acalabrutinib by 2022, according to the Cortellis database from Clarivate Analytics. BeiGene is also vying for a slice of this oncology market, with its phase III candidate BGB-3111.

But drug developers are also increasingly interested in the potential for BTK inhibitors in autoimmune conditions, because the kinase plays a key role in B cell maturation, differentiation and proliferation. AstraZeneca's acalabrutinib, Bristol-Myers Squibb's BMS-986142, Eli Lilly's LY333764, Merck KGaA's evobrutinib and Roche's RG7845 are all in phase II development for rheumatoid arthritis. Merck and Roche are testing their candidates in phase II trials for lupus. Merck is trialling its drug in multiple sclerosis. And in November, Sanofi partnered with Principia Biopharma in a $\$ 765$ million biodollar deal to develop the phase I BTK inhibitor PRN2246 for multiple sclerosis. Asher Mullard 